\title{
La voz del alumnado en la Asociación ApS-(U)CA. Principios de una experiencia
}

\author{
Ana Zarzuela Castro, Esther González Souto, Pablo Calle De los Santos, \\ Esther Carrasquilla Hernández \\ Universidad de Cádiz, Asociación de Aprendizaje y servicio en la Universidad de \\ Cádiz
}

\section{Resumen}

En este artículo se pretende visibilizar una experiencia que está naciendo en relación con el desarrollo de la voz del alumnado en el aprendizaje servicio. Se relatan las vivencias y se formula una propuesta de acción orientada hacia el empoderamiento de la voz del alumnado en el aprendizaje servicio solidario a través de una organización sistemática del movimiento estudiantil. Se parte de la necesidad de movilizar al alumnado de la Universidad de Cádiz, como primer paso para animar este hecho en diferentes universidades, con la meta de compartir sus experiencias y vivencias, para organizar equipos de trabajos heterogéneos. La finalidad de esta propuesta es empoderar la voz del alumnado en diferentes espacios en los que se comparte, dialoga y se tome decisiones sobre el aprendizaje servicio solidario .

\section{Palabras clave}

Aprendizaje servicio solidario, movimiento estudiantil, voz del alumnado.

Fecha de recepción: 210/XII/2015

Fecha de aceptación: 14/IV/2016 


\title{
Reflection and learning service
}

\begin{abstract}
In this article we try to visualize an experience that is emerging in relation to the development of students' voice at service learning. Experiences are told and measures are proposed targeting the empowerment of students voice at service learning through a systematic organization of the students' movement. It starts from the need to mobilize the students at Cadiz University, as a first step to encourage this in different universities, with the goal to share their experiences, to organize heterogeneous working teams. The aim of this proposal is to empower the students voice in different spaces, sharing dialogues and making decisions about service learning.
\end{abstract}

\section{Keywords}

Service learning, students movement, students voice. 


\section{Introducción}

Esta propuesta es una iniciativa que parte del alumnado vinculado a un proceso de aprendizaje servicio solidario (ApS) que se produce en la Asociación de Aprendizaje y Servicio en la Universidad de Cádiz ApS(U)CA en el curso 2015/2016. El interés en el desarrollo de esta iniciativa se asienta en promover la responsabilidad social y compromiso ético de la misma. Como señalan García y Cotrina $(2015,2)$ "es una apuesta por construir y divulgar conocimientos desde un proceso de abajo arriba, desde quienes participan de la lógica y la vivencia del Aprendizaje y Servicio, desde la voz, fuera cual fuera el registro de la misma, de quienes quisieran... participar de este proyecto convertido así en empeño colectivo."

Es decir, se trata de hacer una apuesta en la que se ponga en acción principios metodológicos vinculados a la educación inclusiva para transformar los contextos en espacios más justos, equitativos y solidarios empoderando la voz del alumnado de la Universidad de Cádiz y de diversas Universidades a través del ApS. Como apunta García $(2013,163)$ "la inclusión implica la capacidad de tener voz, pero sobre todo, el reconocimiento del derecho de decidir. Negar esta posibilidad sigue siendo una forma de opresión".

Esta propuesta surge de la necesidad detectada por tres alumnas y un alumno de la Universidad de Cádiz tras acudir al VI Congreso Nacional y I Internacional de Aprendizaje y Servicio Universitario el pasado 28, 29 y 30 de Mayo de 2015 en la Universidad de Granada, donde se puso de evidencia la escasa participación de alumnado frente a la participación muy activa de profesorado. Este equipo tuvo la oportunidad, no sólo de asistir a dicho congreso, sino de participar activamente en él, en espacios destinados para la toma de decisiones conjuntas y en talleres propuestos allí mismo, y con ello pudimos empoderar la voz del alumnado en este aspecto. 
El empoderamiento consiste no solo en tomar la voz, sino en ser tenido en cuenta.

Todo ello llevó a poner en marcha esta propuesta de visibilizar la experiencia del alumnado y la iniciativa de crear un equipo promotor de ApS a través de la voz del alumnado vinculado a la Asociación ApS-(U)CA.

\section{Marco teórico}

¿Qué es el aprendizaje y servicio solidario? ¿se consigue a través de éste empoderar la voz del alumnado? ¿es una metodología emancipadora?

Como indican Puig, Batlle, Bosch y Palos, $(2006,20)$ "es una propuesta educativa que combina procesos de aprendizaje y servicio a la comunidad en un único proyecto bien articulado en el que los participantes aprenden a la vez que trabajan en las necesidades reales del entorno con la finalidad de mejorarlo". Apunta Puig $(2009$,$) "a través del aprendizaje y$ servicio se puede vislumbrar claramente ingredientes de acción inclusiva, orientada desde valores solidarios, donde todos los participantes son igual de importantes y existe espacio para la voz de todos y todas".

Por todo ello, podemos decir que el ApS es una pedagogía libertadora e innovadora siendo su práctica crítica y transformadora. Es una cuestión del presente, pues resuelve problemas inmediatos centrando su mirada en el contexto para que éste sea estimulante y proyecte a ciudadanos críticos, tolerantes, solidarios que busquen la libertad en base al amor y la felicidad de todos.

Aunque los orígenes del ApS se remontan a postulados de James y Dewey (Puig y Palos, 2006) y en concreto, en el voluntariado y en el desarrollo de actividades asociadas a proporcionar un servicio a la comunidad, no se trata de una acción de voluntariado, ya que es un proceso bien definido. En él, el aprendizaje trabaja conjuntamente con la realidad 


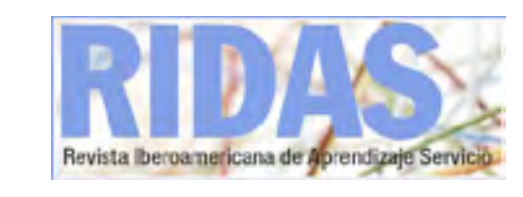

del contexto al que pertenece, ofreciendo recursos y herramientas a todo el colectivo (sea alumnado, profesorado u organizaciones externas al centro conocidas como las alianzas del proyecto) con el fin de una construcción diversa de conocimientos y propia de cada colectivo. Esto supone la constitución de toda una comunidad educativa, con todo lo que su nombre conlleva, y el ApS consigue involucrarla en su aprendizaje. Así pues, "la enseñanza es y ha de ser una actividad de naturaleza cooperativa y solidaria entre los diversos agentes sociales a quienes la elaboración del proyecto les permitirá retomar la voz en el acallado espacio escolar (...) adoptar soluciones adecuadas con las que se deberían sentir comprometidos $y$, en consecuencia, corresponsables" (Beltran y San Martín, 2002, 101).

Pero la innovación va más allá de la metodología, transmitiendo su influencia a la estructura que no es entendida de manera lineal, sino en espiral. Su proceso es el siguiente: análisis social de un entorno real y detección de sus necesidades, propuesta de ideas, planificación de la acción, intervención en el entorno, servicio a la comunidad, evaluación y celebración. Es decir, es un proyecto de investigación transformador, por lo tanto el ApS es una metodología emancipadora $y$, por lo que se puede observar, se interrelaciona de manera directa con la educación inclusiva, pues proyecta a ciudadanos críticos, solidarios, libres y con voz. Como señala Batlle $(2011$,$) el ApS$ "evidencia que los cambios son posibles" a la vez que ofrece una "primera oportunidad para tocar el cambio".

Una experiencia de la Universidad de Cádiz que está empoderada y liderada por los propios estudiantes es el "Proyecto Smile". Es importante resaltar que en este caso estamos hablando de un voluntariado, no un proceso de aprendizaje servicio solidario, pero es un trabajo que ha sido promovido por el alumnado de la Facultad de Medicina de la 


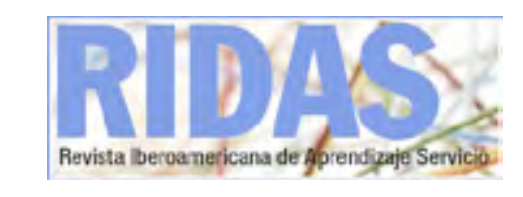

Universidad de Cádiz encauzado por la Asociación de Estudiantes de Medicina de Cádiz. A través de esta entidad, se busca la captación de estudiantes voluntarios, de la titulación que sea, que quieran participar en la animación hospitalaria para hacer más amena la estancia de los niños que están ingresados. En definitiva, defienden la "Medicina de la Risa" para hacer frente a las situaciones en las que se encuentran estos pequeños. Es un voluntariado valioso, pero no está vinculado explícitamente a un proceso de aprendizaje sistemático, intra o extracurricular; por tanto, tampoco existe ese vínculo indisoluble entre aprendizaje y servicio.

Pero una vez más, vemos necesario hacer hincapié que el ApS es una experiencia que va más allá del mero voluntariado, que en sí, nos enriquecería muchísimo. Por ello, consideramos que es necesario resaltar aquellas otras experiencias que van en esta línea de empoderamiento de la voz del alumnado y que se llevan a cabo en la Universidad de Cádiz. Se ha de decir que a través de la dinamización del alumnado bajo filosofías diversas, como es el caso del ApS, se impregnan los curricula del grado de Magisterio de Educación Infantil y Educación Primaria, así como partes del máster Universitario en Profesorado de Educación Secundaria Obligatoria y Bachillerato, Formación Profesional y Enseñanza de Idiomas y el máster Interuniversitario en Educador Ambiental. Asimismo, recientemente en el 2015 se institucionaliza la Asociación ApS(U)CA abriéndose así nuevos paradigmas pedagógicos y sociales.

\section{Propuesta}

Por todo ello, nace esta propuesta que está dando sus primeros pasos en el Campus de Puerto Real de la Universidad de Cádiz, concretamente a través de la Asociación ApS - (U)CA dinamizado por tres alumnas y un alumno de la misma, pues detectaron la necesidad de construir 
conjuntamente espacios de diálogo, reflexión y toma de decisiones sobre Aps.

La finalidad de esta propuesta será empoderar la voz del alumnado a través del movimiento estudiantil del Estado Español para así poder compartir experiencias, vivencias, decisiones sobre el ApS y tener la posibilidad de crear equipos de trabajo heterogéneos.

Todo esto se dará a través de diferentes fases que se desarrollarán de forma paulatina y sistemática para así asegurar el éxito en todo el proceso. En primer lugar se está creando un equipo promotor, el cual estará dinamizado por esos cuatro alumnos mencionados, que serán los responsables de procurar esa unión de voces dentro de la propia Universidad de Cádiz. Posteriormente tendrá lugar la segunda fase en la que se comenzarán a crear alianzas con otras asociaciones y universidades del Estado Español poniéndonos en contacto con sus respectivos equipos promotores. $Y$ finalmente, en una tercera fase, comenzaremos a trabajar de forma conjunta, en todos los aspectos anteriormente mencionados, a través de la creación de equipos heterogéneos en los que participen todo el alumnado de las diferentes universidades, asociaciones, entidades y colectivos.

A continuación, presentamos una figura en la que se exponen las relaciones que intervienen en el proceso.

Figura 1. Comunidad implicada en la experiencia

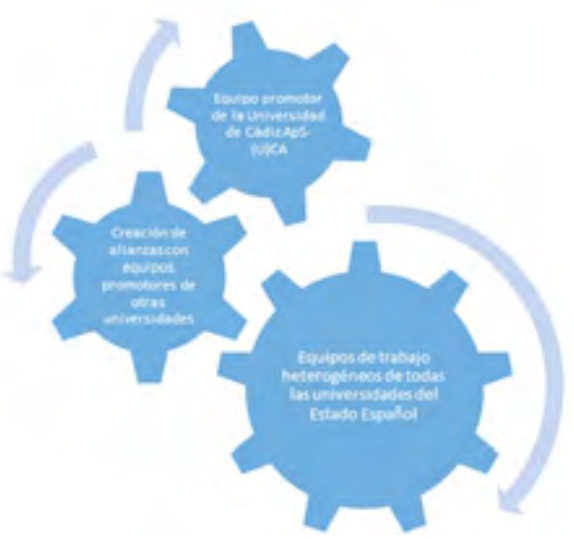

Fuente: elaboración propia 
Un aspecto del que no debemos olvidarnos en ningún momento durante todo el desarrollo de estas fases, será el realizar un seguimiento del mismo a través de la reflexión continua, pues sin la cual estaríamos perdiendo la misma esencia del ApS en el camino, $y$ es algo que no pretendemos en ningún momento.

Actualmente nos encontramos aún en la primera fase de esta propuesta. Estamos creando ese equipo promotor dentro de la Universidad de Cádiz, dinamizado por cuatro estudiantes de la Asociación ApS(U)CA. Estos realizarán el acompañamiento, durante todo el proceso, a todo el alumnado de la Universidad de Cádiz que se incorpore a esta aventura y facilitará la creación de alianzas, aunque por supuesto cualquier alumno participará activamente durante todo el proceso sin que tengan que ser estos estudiantes quienes realicen todos los movimientos.

Además la institucionalización de la
Asociación ApS-(U)CA se creó para acompañar procesos de ApS en diversos espacios, no solo universitarios, para así poder atender las necesidades de todos los contextos y se ha de comentar que para el próximo año 2016-2017 nos embarcamos en propuestas, tejiendo una construcción conjunta con diferentes entidades, asociaciones $y$ colectivos. Seguidamente presentamos una figura de esta primera fase.

Figura 2. Participantes de la primera fase

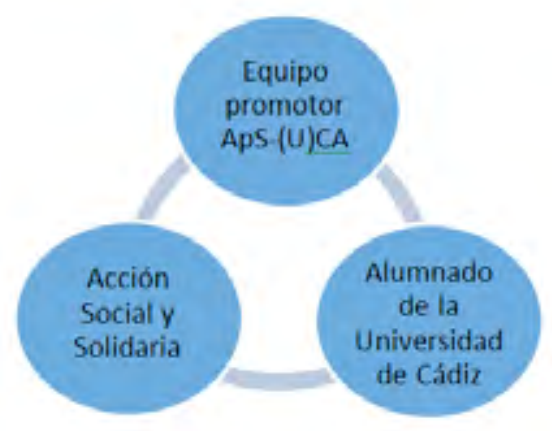

Fuente: elaboración propia

El servicio que se pretende ofrecer es proporcionar un espacio de encuentro 


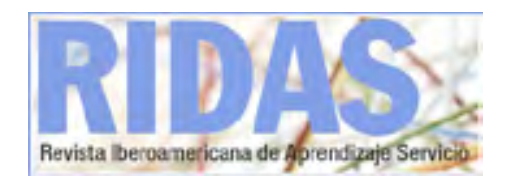

estudiantil de alumnos de la universidad de Cádiz y de diferentes universidades, colectivos, asociaciones y entidades de distintos ámbitos. De esta forma se intenta promover la esencia del ApS a través de las voces de todos. Para ello, el primer encuentro se pretende organizar en el Campus de Puerto Real de la Universidad de Cádiz (UCA) junto con la colaboración de Acción Social y Solidaria de la UCA.

\section{Estado de la experiencia}

Como vemos, esta experiencia se encuentra en sus momentos de inicio y está comenzando a arrancar. Aún se están dando los primeros pasos de esa fase 1 , ya que el equipo promotor está terminando de fundar sus bases y planificar la captación del alumnado interesado en participar en este camino del despertar de la voz del alumnado en el ApS. Nada más comenzar con esta aventura, consideraron que era necesario darse a conocer como equipo y no como personas que trabajan individualmente; por ello, crearon una dirección de correo electrónico conjunta que les permite contactar y ser contactados por diversos contextos y abordar muchas de las actividades que desarrollan.

Tabla 1. ¿Cómo nos organizamos? Reuniones

\begin{tabular}{|c|c|c|c|}
\hline \multicolumn{4}{|c|}{$\begin{array}{l}\text { ¿CÓNOO NOS ORCANIZAMCS? } \\
\text { REUNIONES }\end{array}$} \\
\hline ¿Cuindo? & Senanales & Cuincenales & Nersuales \\
\hline ¿Quieines? & $\begin{array}{l}\text { Equipo promotor } \\
\text { ApS-(U)CA }\end{array}$ & $\begin{array}{l}\text { Acompenante. } \\
\text { Docente UCA }\end{array}$ & $\begin{array}{l}\text { Asambles } \\
\text { Auociación Aos. } \\
\text { (UICA }\end{array}$ \\
\hline ¿Cóms? & Shype & $\begin{array}{l}\text { Reunionas } \\
\text { pretenciales }\end{array}$ & $\begin{array}{l}\text { Reuniones } \\
\text { prectencialer }\end{array}$ \\
\hline ¿Para quê? & $\begin{array}{l}\text { Estructura } \\
\text { orgarizetivo } \\
\text { Toms de } \\
\text { declisones }\end{array}$ & $\begin{array}{l}\text { Formatión * } \\
\text { atompar̆amiento }\end{array}$ & $\begin{array}{c}\text { Eitructura } \\
\text { organizativa } \\
\text { Tons de } \\
\text { declisiones }\end{array}$ \\
\hline Instrumentos & Chario & ctus + Blog + Hede & ciales \\
\hline
\end{tabular}

Fuente: elaboración propia

Para que todo esto sea posible, los cuatro estudiantes que dinamizan el proceso se están coordinando y tomando decisiones de forma conjunta a través de reuniones semanales haciendo uso de redes sociales como Skype, pues en estos momentos se encuentran en espacios diferentes de Andalucía. Los roles que se asumen en dichos encuentros van rotando semanalmente de forma que la función no radique en una única persona, potenciado así habilidades 
diversas para todos. Las decisiones que se toman en dichas reuniones $y$ los acuerdos a los que llegan, quedan recogidos en actas, que se trabajan a través de aplicaciones como Google Drive, para facilitar el acceso de todos y de esta forma tener un seguimiento de todo lo que se está abordando en las reuniones, utilizándose como mecanismo de reflexión de mejora sobre la práctica. Además, se utilizará la misma aplicación para trabajar tanto de forma individual, elaborando diarios, como grupal, a través de actas. Ambos son de necesidad puesto que, aunque en las actas se recogen aquellos aspectos que han de tratarse y a los acuerdos que hemos llegado de manera conjunta; los diarios son utilizados como una forma de expresarse individualmente, de cómo sentimos, nos emocionamos y vivimos cada uno de los pasos que damos en el avance de nuestra promotora asociación, aunque finalmente se vayan a compartir.

Paralelamente a estas reuniones se realizan otras de forma quincenal con una docente de la Universidad de Cádiz, la cual es pionera en aprendizaje y servicio solidario. Con ello se pretende profundizar en esa formación necesaria para poder abordar todos los proyectos que se están desarrollando y aprender sobre diversas metodologías, así como realizar tertulias dialógicas que permitan el enriquecimiento personal y profesional de todos los implicados en este proceso. A esto lo llamamos andamiaje pedagógico siguiendo los postulados vigostkyanos.

Asimismo, es muy importante resaltar que una de las primeras decisiones que se tomaron para dar a conocer esta iniciativa fue crear un blog, donde se cuentan las experiencias que se están desarrollando en ApS, aquellas otras que aunque no sean ApS sí ayudan a la formación y están relacionadas con la innovación, las inquietudes que van teniendo, comparten información básica que van teniendo sobre la asociación ApS(U)CA, además de ir visibilizando todos los caminos que vamos 
realizando en todo el proceso. Una vez creado el blog, era necesario darlo a conocer, pues inicialmente tenía poco movimiento al ser desconocido. Con el fin de iniciar esa difusión se decidió acceder a redes sociales, como Twitter y Facebook, donde llegaron a muchas personas de diferentes puntos del país, e incluso fuera de él, dando como consecuencia la visibilización del trabajo que estamos llevando a cabo.

Pero toda esa difusión vino de la mano de la autogestión. Para mejorar la formación de los cuatro estudiantes en cuestión y así dar a conocer el ApS en más espacios y compartir sus experiencias, se experimentó la necesidad de contar con un fondo monetario que permitiese mayor libertad económica para poder abordar estas cuestiones. Por ello, decidieron crear chapas, relacionadas con la temática del ApS, y continuar así con la difusión de todas las actividades en las que están implicados y al mismo tiempo de disponer de fondos que permitan cubrir las necesidades de formación. Todo esto les llevó a ponerse en contacto con el Colectivo CEPA de Cádiz, el cual es responsable de la prevención e inserción social, quienes se encargaron de realizar las chapas a partir de los diseños elaborados por estos cuatro estudiantes. Ese acercamiento ha provocado que CEPA se interese también por todo aquello que se está abordando a través de la voz del alumnado y quieran participar conjuntamente en futuros proyecto, posibilitando la vinculación con nuevas alianzas.

Por otra parte, se están llevando a la práctica diversos proyectos en los que la voz del alumnado es más que importante. Dentro de estos proyectos, encontramos procesos de aprendizaje y servicio solidario, voluntariados y participación en diversos encuentros.

- Procesos de aprendizaje y servicio solidario (ApS) 
Figura 3. Procesos de ApS

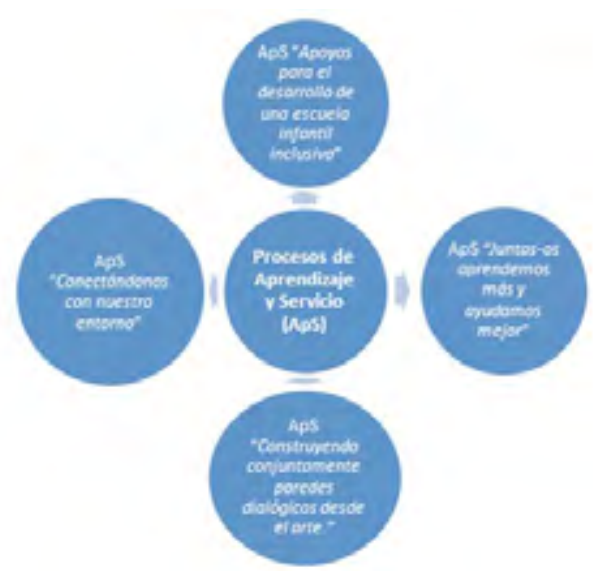

Fuente: elaboración propia

La voz del alumnado es algo fundamental en todos los procesos de enseñanza-aprendizaje, y si nos estamos refiriendo a un ApS, esa voz toma una mayor importancia. Escuchar las necesidades de aquellos que participan en el ApS, tener en cuenta sus inquietudes, intereses, miedos, prestar atención a las propuestas que surgen y a la creación de alianzas... En definitiva, aspectos fundamentales que sin escuchar las voces del alumnado, que son los implicados directos a todo esto, harían que el ApS no siguiese adelante.

- Voluntariado.

Figura 4. Actividades de voluntariado

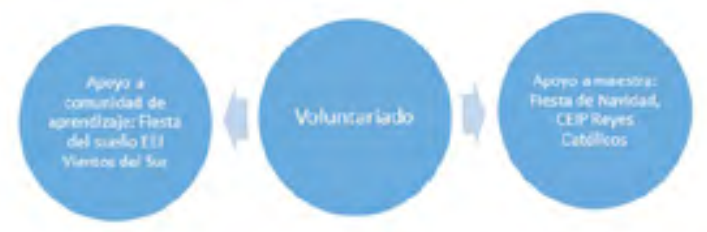

Fuente: elaboración propia

Aunque no se tratan de procesos de ApS en sí mismo, estos voluntariados sí que se encuentran vinculados a algunos de los ApS anteriormente comentados. Unos como primera toma de contacto con el centro y otros como resultado de esa relación previamente establecida en el ApS.

- Participación en encuentros. 


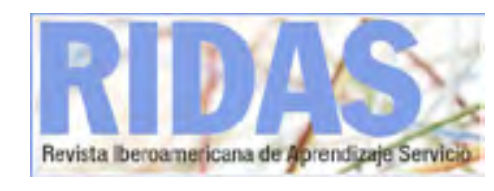

Figura 5. Participación en encuentros

Figura 6. Actividades de aprendizaje y servicio

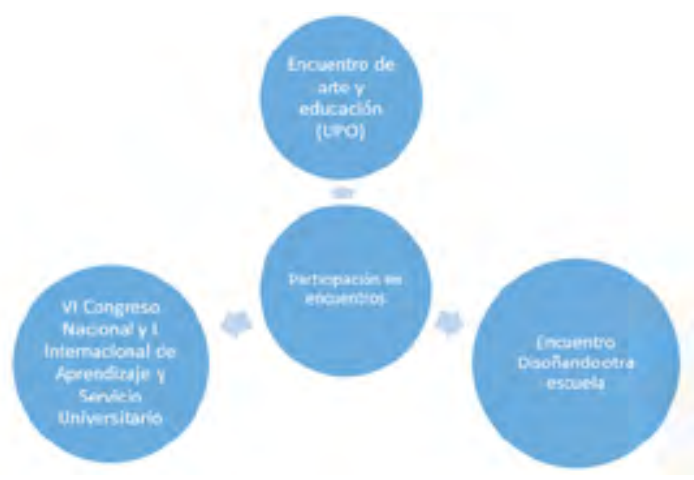

Fuente: elaboración propia

Las experiencias en ApS se han dado a conocer en diferentes encuentros. Algunos de ellos bajo la temática del aprendizaje y servicio solidario, como es el congreso, y otros como modelo de innovación educativa, para así darlo a conocer entre aquellos que aún no están familiarizados con esta filosofía pedagógica. solidario



Fuente: elaboración propia

\section{Resultados}

Como ya hemos comentado, la concreción de la propuesta está en proceso, puesto que es una construcción conjunta entre todas las personas implicadas. Aún así, sentíamos la necesidad de compartir en este espacio el proceso de esta iniciativa para que se vaya sintiendo el camino que estamos recorriendo conjuntamente y cada vez se unan más las sinergias de todos. Esto es así, ya que estamos abiertos a propuestas, iniciativas, sugerencias e incertidumbre que pongan en desequilibrio ideas preconcebidas 
para la construcción de bases sólidas del movimiento estudiantil y conseguir una mejor formación. De esta forma, perseguimos la formación continua que nos permite renovarnos $y$ ser mejores profesionales, promoviendo que el conocimiento no sea estático y se construya conjuntamente nuestro conocimiento y con ello una mejor sociedad.

\section{Conclusiones}

En todo este proceso, y lo que se lleva realizado de él hasta el momento, despierta en estos cuatro estudiantes una gran variedad de emociones y sentimientos dada la gran cantidad de iniciativas y proyectos que se están abordando. Analicemos detenidamente cada una de las partes que se ven en juego en esta iniciativa.

A nivel profesional, dinamizado por el alumnado, supondrá un gran reto en el que se realizará un aprendizaje bidireccional entre todos los agentes implicados. Además, abrirá otras oportunidades que antes no habían experimentado, cómo es construir conjuntamente espacios para alumnado de diversas universidades coordinando diferentes colectivos, entidades, instituciones, movilizando los recursos de manera justa y equitativa para transformar conjuntamente el entorno educativo y por consiguiente social. Ante esta perspectiva están emocionados, ya que les permitirá crear vínculos con otros estudiantes y unir fuerzas para conseguir esta transformación que tanto se está persiguiendo; pero al mismo tiempo también despierta algo de incertidumbre puesto que se arriesgan y afrontan el reto de una iniciativa muy ambiciosa en el que pretenden poner en acción sueños, cómo es el de que otros estudiantes se interesen en acompañarlos en esta travesía.

A nivel personal, todos los miembros de esta iniciativa nos embarcamos en una gran aventura en la que nos genera una gran ilusión, entusiasmo e incertidumbre, pues apostamos por construir conjuntamente espacios 
más justos, equitativos, libres, solidarios y estimulantes, en el que la voz del alumnado sea esencial para promover una transformación social y educativa. Están muy contentos de ver que, lo que se lleva hecho hasta el momento, pues está teniendo muy buena acogida y que cada vez son más las personas quienes siguen la pista de los proyectos en los que estamos involucrados. Pero hasta llegar a ese sentimiento han pasado por momentos de desequilibrio tan necesarios para el desarrollo tanto personal como profesional de cada uno de ellos. Se ha de decir que estos resultados gratificantes no se vieron nada más empezar y se ha estado caminando en el anonimato durante un tiempo. Por suerte todo esto va cambiando poco a poco y se empieza a ver la luz tras esos pequeños momentos de oscuridad.

Para finalizar queremos hacer visible la importancia de empoderar la voz del alumnado en el aprendizaje y servicio solidario para que éste se erija en el auténtico protagonista del mismo.

\section{Referencias bibliográficas}

Batlle, R. (2011). ¿De qué hablamos cuando hablamos de aprendizaje y servicio. Revista Crítica, 972, 1-16.

García, M. (2013) La cooperación internacional para el desarrollo de la Educación Inclusiva. Algunas contradicciones en el caso de EspañaLationoamérica. Revista de Educación inclusiva, 5 (1), 160-173.

García, M. y Cotrina, M (2015). Aprendizaje y Servicio en la formación del profesorado: Haciendo efectiva la responsabilidad social y el compromiso ético. Profesorado: Revista de currículum y formación del profesorado 19 (1), 1-6.

Puig, J. M.a (Coord.) (2009). Aprendizaje servicio (ApS). Educación y compromiso cívico. Barcelona: Graó.

Puig, J.M.; Batlle, R. Bosch, C. y Palos, J. ( 2006 ). Aprendizaje 
Servicio. Educar para la ciudadanía. Barcelona: Octaedro.
Puig, J.M. y Palos, J. (2006). Rasgos pedagógicos del aprendizaje servicio. Cuadernos de Pedagogía. 60-66. 